Digilec 8 (2021), pp. 53-65

Fecha de recepción: 02/09/2021

Fecha de aceptación: 05/10/2021

DOI: https://doi.org/10.17979/digilec.2021.8.0.8634

(c) (1) (?)

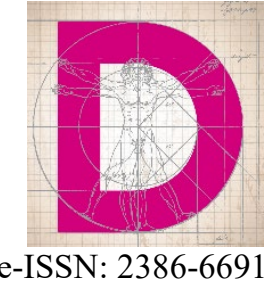

\title{
A TRAVÉS DEL ESPEJO: BENEFICIOS DE LAS LESSON STUDY PARA PROFESORADO Y ALUMNADO DE EDUCACIÓN OBLIGATORIA
}

\author{
THROUGH THE LOOKING GLASS: BENEFITS OF THE LESSON \\ STUDY FOR COMPULSORY EDUCATION TEACHERS AND \\ STUDENTS
}

María Elena GÓMEZ-PARRA*

Universidad de Córdoba

Orcid: http://orcid.org/0000-0001-7870-3505

\section{Resumen}

La literatura sobre aprendizaje cooperativo se muestra unánime en las ventajas que este ofrece para el desarrollo de habilidades esenciales para el ciudadano del siglo XXI. Las Lesson Study (LS) son "not only a means of improving the skills and knowledge of teachers, but also a way to improve the knowledge base of the teaching profession" (Stigler, 2004: ix). Los dos objetivos principales de este trabajo son: i. Analizar y comparar el impacto de dos rondas de implementación de LS (175 estudiantes) en el marco del proyecto Dispositifs inclusifs de cooperation - DICO+ (2018-1-FR01-KA201047904); y ii. Analizar el impacto formativo de las LS entre el profesorado. Se usó la metodología de análisis de métodos mixtos para esta investigación. Los resultados cuantitativos revelaron interesantes comparaciones sobre las preferencias del alumnado cuando trabajan bajo el paradigma de las LS, mientras que los datos cualitativos indican que las LS han contribuido significativamente a la mejora de la formación y a la praxis docente del profesorado. Nuestras conclusiones indican que el alumnado reconoce los beneficios sociales de las LS y apuntan a que el profesorado debería conocerlas más a fondo e implementarlas con frecuencia, porque la estructuración del trabajo cooperativo previo a la implementación beneficia el aprendizaje (Sulfemi \& Kamalia, 2020) y, además, puede contribuir a mejorar la formación (inicial y permanente) del profesorado, así como a mejorar su disposición para adoptar técnicas adaptativas en el aula.

Palabras clave: lesson Study; aprendizaje cooperativo; formación del professorado; proyecto internacional; educación obligatoria.

* Facultad de Ciencias de la Educación. Avda. San Alberto Magno, s/n. 14071 Córdoba (España). Email: elena.gomez@uco.es 


\begin{abstract}
The literature on cooperative learning is unanimous in the advantages it offers for the development of essential skills for the $21^{\text {st }}$ century citizen. Lesson Studies (LS) are "not only a means of improving the skills and knowledge of teachers, but also a way to improve the knowledge base of the teaching profession" (Stigler, 2004: ix). The two main objectives of this study are: $i$. To analyse and compare the impact of two rounds of LS implementation (175 students) in the framework of the project Dispositifs Inclusifs de Cooperation - DICO+ (2018-1-FR01-KA201-047904); and ii. To analyse the impact on training of LS among teaching staff. The Mixed Methods Research approach was used for this analysis. The quantitative results of this study revealed interesting comparisons of students' preferences when working under the LS paradigm, while the qualitative data indicate that the LS has contributed significantly to the improvement of teachers' training and teaching practice. Our conclusions indicate that students recognize the social benefits of the implementation of the LS, and suggest that teachers should learn more about and implement the LS more often, because structuring cooperative work prior to implementation benefits learning (Sulfemi \& Kamalia, 2020) and can contribute to improving teachers' training (both pre- and in-service), as well as their readiness to adopt adaptive techniques in the classroom.
\end{abstract}

Key Words: lesson Study; cooperative learning; teacher training; international project; compulsory education. 


\section{INTRODUCCIÓN}

La literatura especializada sobre aprendizaje cooperativo (AC) se muestra unánime en las ventajas que este ofrece para el desarrollo de habilidades esenciales para el ciudadano del siglo XXI, tales como autonomía (Shi \& Han, 2019), responsabilidad (Widita \& Nurihsan, 2020) o el trabajo en equipo (Kyprianidou, Demetriadis, Tsiatsos, \& Pombortsis, 2012), entre otras. Las conocidas como Lesson Study (LS) son concebidas como "not only a means of improving the skills and knowledge of teachers, but also a way to improve the knowledge base of the teaching profession" (Stigler, 2004: ix). Por tanto, el valor pedagógico de las LS se estima no solo en las oportunidades educativas que presenta para el alumnado, sino también en sus cualidades para la formación del profesorado en sus dos modalidades: inicial y permanente.

Las LS nacieron en Japón como un método de enseñanza basado en un enfoque abierto. Estrella et al. (2020) las identifican como una constante en la forma en la que los profesores japoneses llevan a cabo sus clases. En la enseñanza japonesa, el profesor comienza con un repaso de la lección anterior y luego presenta el problema del día en forma de pregunta. Los alumnos deben comprender el problema y trabajar (generalmente en grupo) para discutir las formas de resolverlo. Por tanto, la discusión y la reflexión se erigen en una parte integral del proceso de aprendizaje. Perry y Lewis (2009: 366) describen las LS poniendo el énfasis en el ciclo instructivo para el profesorado, que constituye un interesante elemento diferenciador en este modelo:

Lesson study is a cycle of instructional improvement in which teachers work together to: formulate goals for student learning and long-term development; collaboratively plan a "research lesson" designed to bring to life these goals; conduct the lesson in a classroom, with one team member teaching and others gathering evidence on student learning and development; reflect on and discuss the evidence gathered during the lesson, using it to improve the lesson, the unit, and instruction more generally; and if desired, teach, observe, and improve the lesson again in one or more additional classrooms (Lewis 2002b).

Wood y Cajkler (2018) describen el proceso de las LS en torno a cinco fases: En primer lugar, un grupo de profesores identifica un reto de aprendizaje que experimentan sus alumnos. A continuación, el grupo docente planifica el desarrollo de una clase (generalmente basada en investigación) para afrontar el reto. En tercer lugar, mientras uno de los miembros del grupo docente imparte la clase, el resto (que pueden ser profesores en formación o en ejercicio) observa el desarrollo de la sesión, poniendo especial énfasis en las reacciones y en el aprendizaje de los alumnos. En cuarto lugar, el grupo docente evalúa la sesión basándose en sus reflexiones y observaciones (que se han llevado a cabo durante el paso anterior). Para el último paso, el grupo se centra en su propio aprendizaje como profesores, revisando el reto y su experiencia. Si hay consenso, el grupo continúa el proceso implementando la sesión revisada en un grupo comparable. En ocasiones, las sesiones se graban, lo que facilita el proceso final de revisión y análisis de la sesión por parte del equipo docente. 
Los beneficios de las LS son reconocidos ampliamente por la literatura del área. Podemos separar (a niveles meramente explicativos) las ventajas que tiene implementar (con cierta asiduidad) este enfoque para el alumnado y para el profesorado. Wahyukti (2017: 291) indica que se trata de "a powerful instructional method to enhance the students' cooperation in terms of positive interdependence, individual accountability, equal participation, simultaneous interaction and social skills". Guner y Akyuz (2020) afirman que el potencial de las LS radica en su capacidad de anticiparse al pensamiento, la comprensión y las reacciones del alumnado. Los resultados del estudio de Hasan, Lukitasari y Ernawati (2021) llevan a estos autores a concluir que la aplicación de las LS mejora el proceso de aprendizaje de los alumnos de forma activa y animada, además de tener un impacto directo en el aumento de su comprensión sobre los conceptos de la materia que han estudiado. Por tanto, podemos afirmar que los hallazgos de la investigación reportan ganancias para el alumnado en distintos planos (por ejemplo, habilidades sociales, de aprendizaje y cognitivas, entre otros).

Como avanzábamos, la implementación de las LS también tiene beneficios para el profesorado. Peña-Trapero y Pérez-Gómez (2017: 66) parten de la resistencia de los docentes al cambio y al progreso en educación. Su trabajo demuestra la potencialidad de las LS "for the transformation of schools in the twenty-first century and the construction of a reflected, shared, emerging pedagogical capital for teachers". A este respecto, Lee y Tan (2020: 13) afirman que "The adoption of Lesson Study involves reculturing that can be challenging”. Schipper, van der Lans, de Vries, Goei y van Veen (2020) entienden que las LS juegan un papel pivotante a la hora de influir en las percepciones del profesorado, haciéndolo más consciente de las diferentes necesidades educativas del alumnado y cómo dirigirse a ellas de manera adecuada. Perry y Lewis (2009) afirman que el desarrollo profesional es la clave para la mejora educativa, para lo que las LS se erigen en un potente instrumento porque, entre otros, suponen una práctica colaborativa entre el profesorado que se puede calificar por ser sostenible, intensa y basada en la práctica. Además, los datos que reporta el sistema educativo japonés hablan a favor de la implementación de este enfoque, que está ampliamente extendido en el país nipón.

\section{OBJETIVOS}

Este trabajo se marca dos objetivos fundamentales, que formulamos de la siguiente manera:

Objetivo 1. Analizar y comparar el impacto de las LS en dos grupos de alumnos de educación obligatoria en España $(n=175)$.

Objetivo 2. Analizar el impacto formativo que tienen las LS entre el profesorado participante $(n=4)$. 


\section{MÉTODO}

\subsection{Contexto y Participantes}

Este estudio se enmarca en el proyecto europeo Dispositifs inclusifs de cooperation - DICO+ (2018-1-FR01-KA201-047904) financiado por la Agencia Nacional Francesa, cuyo objetivo fundamental es analizar el impacto de las LS a nivel internacional, y comprobar si su implementación influye de manera positiva en la reducción del abandono escolar. La población meta de este proyecto son alumnos de educación obligatoria, de entre 8 y 14 años, provenientes de 8 instituciones localizadas en 7 países europeos: Francia, España, Países Bajos, Italia, Hungría, Lituania y Rumanía.

Este trabajo analizará los datos de dos rondas de implementación en el contexto español, que se llevaron a cabo durante los cursos académicos 2018-2019 y 2020-2021 con dos grupos diferenciados de participantes. Por un lado, identificamos un grupo de alumnos (Grupo 1), que formaron parte de las dos implementaciones descritas, mediando entre ellas el curso 2019-2020, donde la pandemia por COVID-19 obligó al alumnado a confinarse, por lo que el proyecto DICO+ decidió suspender las implementaciones en el aula. Por otro lado, identificamos el grupo de profesores (Grupo 2) que formaron parte de estas implementaciones. Pasamos a describir en detalle ambos grupos:

Grupo 1: 175 alumnos provenientes de 2 etapas educativas (Ed. Primaria y Ed. Secundaria), con quienes se implementan las LS en dos asignaturas diferentes.

- Educación Primaria (curso 2018-2019). Asignatura: inglés. Curso: $3^{\circ}$ de Educación Primaria. Número de alumnos: 48.

- Educación Primaria (curso 2020-2021). Asignatura: inglés. Curso: $3^{\text {o }}$ de Educación Primaria. Número de alumnos: 42.

- Educación Secundaria (curso 2018-2019). Asignatura: matemáticas. Curso $1^{\circ}$ ESO. Número de alumnos: 19.

- Educación Secundaria (curso 2018-2019). Asignatura: matemáticas. Curso: $1^{\circ}$ ESO. Número de alumnos: 33.

- Educación Secundaria (curso 2020-2021). Asignatura: matemáticas. Curso: $1^{\circ}$ ESO. Número de alumnos: 33 .

Grupo 2: 4 profesores (en formación y en ejercicio), que intervinieron en las dos implementaciones de las LS durante los dos cursos académicos señalados.

- Curso académico 2018-2019: 2 profesores de la asignatura de inglés.

- Curso académico 2020-2021: 2 profesores, de los cuales uno pertenece al área de inglés y el otro a la de matemáticas.

\subsection{Instrumentos}

Se han diseñado dos instrumentos para la recopilación de datos cuantitativos y cualitativos, que se pasan a describir. 
Para la recolección de datos cuantitativos, el proyecto DICO+ diseñó y validó mediante método Delphi un cuestionario de 13 preguntas de respuesta de opción múltiple o dicotómica en inglés. Este cuestionario se tradujo al español y se volvió a validar mediante Delphi, arrojando un alfa de Cronbach de .775 y una omega de McDonald de .781 , lo cual indica una validez y confianza de la escala muy altas, según Campo Arias y Oviedo (2008).

Este trabajo analizará las respuestas del Grupo $1(\mathrm{n}=175)$ a las preguntas número 3 y 4 de dicho cuestionario para las dos implementaciones de las LS, que responden a las preferencias del alumnado para el trabajo en el aula y analizan sus gustos en las actividades que realizan cuando trabajan en grupo (por ejemplo, si les gusta ayudar a otros a aprender o si les gusta charlar con sus compañeros sobre la tarea que se les ha encomendado). Los datos demográficos de este grupo son $46 \%$ chicos y $54 \%$ chicas, todos de nacionalidad española.

Por otro lado, el instrumento diseñado para la recogida de datos cualitativos para el Grupo 2 de este estudio (es decir, profesorado) fue una entrevista estructurada en inglés en la que los participantes, una vez introducidos sus datos personales (sexo y edad), aportaron sus respuestas a las siguientes preguntas:

1. Number of teaching experience years.

2. Languages you speak.

3. Did you know about Lesson Study before participating in $\mathrm{DICO}+$ ?

4. Did you know about Cooperative Learning before participating in $\mathrm{DICO}+$ ?

5. Have you ever implemented Cooperative Learning techniques in your classroom?

6. If you answered 'Yes' to question no. 5, can you say if you think Cooperative Learning was effective and in which ways it was so? If you answered 'Not' to question no. 5 , can you say why it was not?

7. Can you say if the implementation of Lesson Study was effective for your students? If 'yes', can you say in which ways it was? If 'not', can you say why it was not?

8. Finally, can you summarize in which ways Lesson Study has had an impact on your own teaching practices and on your own training as a teacher?

Los participantes del Grupo 2 de este estudio $(n=4)$ ofrecieron sus respuestas de manera escrita (mediante archivo adjunto a un correo electrónico). De los 4 profesores, 3 son mujeres y 1 es hombre, todos de nacionalidad española. Este trabajo analizará las respuestas a las preguntas número 3, 4 y 8 , que ofrecen información sobre su conocimiento previo sobre las LS y el AC, así como sobre el impacto que la implementación de las LS y su participación en DICO+ han tenido en su formación como docentes y en sus prácticas de aula.

\subsection{Procedimiento}

Este estudio sigue un método de investigación mixta que analiza dos conjuntos de datos. Para el bloque de datos que emanan de las respuestas del alumnado (Grupo 1, $\mathrm{n}=$ 
175) a nuestro cuestionario, realizaremos un análisis cuantitativo de los datos mediante Jamovi (v. 1.2.5.0). Para el bloque de datos proveniente del profesorado (Grupo 2, $\mathrm{n}=4$ ), realizaremos un análisis de contenido siguiendo el modelo de la Teoría Fundamentada (Creswell \& Poth, 2018: 315-316), que estos autores definen como: "a qualitative research design in which the inquirer generates a general explanation (a theory) of a process, an action, or an interaction".

\section{RESULTADOS}

Dividiremos el análisis de los resultados de esta investigación en dos bloques, que contendrán los datos cuantitativos y cualitativos de este trabajo.

\subsection{Resultados grupo 1: análisis cuantitativo}

El análisis de las respuestas de los participantes $(\mathrm{n}=175)$ a la pregunta no. 3 (“Te gusta trabajar en clase con tus compañeros/as (por ejemplo, trabajar en grupos o por parejas)?”) para las dos implementaciones de las LS (curso académico 2018-2019 y curso académico 2020-2021) se resume en la Tabla 1 a continuación:

\section{Tabla 1}

Resultados cuantitativos pregunta 3 (Grupo 1)

\begin{tabular}{|l|c|c|c|c|c|c|c|c|}
\cline { 2 - 9 } \multicolumn{1}{c|}{} & \multicolumn{2}{c|}{ Nunca } & \multicolumn{2}{c|}{ A veces } & \multicolumn{2}{c|}{ Normalmente } & \multicolumn{2}{c|}{ Siempre } \\
\cline { 2 - 9 } \multicolumn{1}{c|}{} & Prim. & Sec. & Prim. & Sec. & Prim. & Sec. & Prim. & Sec. \\
\hline $\begin{array}{l}\text { Curso 18-19 } \\
(\mathrm{n}=100)\end{array}$ & $1 \%$ & - & $9 \%$ & $12 \%$ & $13 \%$ & $14 \%$ & $24 \%$ & $27 \%$ \\
\hline $\begin{array}{l}\text { Curso 20-21 } \\
(\mathrm{n}=75)\end{array}$ & - & - & $7 \%$ & $4 \%$ & $16,34 \%$ & $14 \%$ & $29,66 \%$ & $29 \%$ \\
\hline
\end{tabular}

Los resultados del análisis de las respuestas a la pregunta no. 4 de nuestro cuestionario (“¿Qué te gusta del trabajo en grupo?”), donde se ofrecían 10 posibles opciones (que se detallan en la Tabla 2, a continuación) y se les pedía que marcasen 4, son los siguientes: 
Tabla 2

Resultados cuantitativos pregunta 4 (Grupo 1)

\begin{tabular}{|c|c|c|c|c|}
\hline & \multicolumn{2}{|c|}{$\begin{array}{l}\text { Curso 18-19 } \\
(\mathrm{n}=100)\end{array}$} & \multicolumn{2}{|c|}{$\begin{array}{l}\text { Curso 20-21 } \\
(\mathrm{n}=75)\end{array}$} \\
\hline & Prim. & Sec. & Prim. & Sec. \\
\hline $\begin{array}{l}\text { Ayudar a otros a aprender } \\
\text { juntos }\end{array}$ & 44 & 35 & 33 & 25 \\
\hline Poder hablar & 8 & 6 & 7 & 7 \\
\hline $\begin{array}{l}\text { Dejar que otros hagan } \\
\text { cosas cuando yo no sé } \\
\text { hacerlas }\end{array}$ & 10 & 14 & 6 & 4 \\
\hline $\begin{array}{l}\text { Dejar que otros hagan } \\
\text { cosas cuando a mí no me } \\
\text { gusta hacerlas }\end{array}$ & 7 & 7 & 2 & 2 \\
\hline $\begin{array}{l}\text { Charlar con mis } \\
\text { compañeros/as sobre el } \\
\text { trabajo que vamos a hacer }\end{array}$ & 37 & 26 & 21 & 18 \\
\hline $\begin{array}{l}\text { Trabajar de forma } \\
\text { diferente a la habitual }\end{array}$ & 26 & 32 & 25 & 21 \\
\hline $\begin{array}{l}\text { Ayudar a mis } \\
\text { compañeros/as a aprender } \\
\text { cosas nuevas }\end{array}$ & 38 & 18 & 33 & 14 \\
\hline $\begin{array}{l}\text { Hacer amigos/as } \\
\text { nuevos/as }\end{array}$ & 19 & 18 & 25 & 18 \\
\hline $\begin{array}{l}\text { Que mis compañeros/as } \\
\text { me ayuden a hacer un } \\
\text { ejercicio, resolver un } \\
\text { problema, etc. }\end{array}$ & 19 & 20 & 11 & 13 \\
\hline $\begin{array}{l}\text { Trabajar con varios/as } \\
\text { compañeros/as sin que } \\
\text { los/as profesores/as } \\
\text { intervengan; así tengo yo } \\
\text { la responsabilidad }\end{array}$ & 8 & 5 & 8 & 4 \\
\hline
\end{tabular}

\subsection{Resultados grupo 2: análisis cualitativo}

Las respuestas de los participantes del Grupo 2 de nuestro estudio $(n=4)$ a la pregunta no. 3 ("Did you know about Lesson Study before participating in DICO+?") son unánimes: el $100 \%$ de los profesores no había oído hablar del enfoque Lesson Study antes de participar en este proyecto. Sin embargo, las respuestas a la pregunta no. 4 ("Did you know about Cooperative Learning before participating in DICO+?”) revelan que el $100 \%$ de los participantes sí conocía el enfoque de Aprendizaje Cooperativo anteriormente, indicando 3 de los 4 participantes que lo habían usado en un aula con anterioridad. 
El análisis de contenido de las respuestas de los profesores participantes (que han sido identificados con la sigla "P" más un número en nuestro análisis cualitativo) a la pregunta no. 8 ("Can you summarize in which ways Lesson Study has had an impact on your own teaching practices and on your own training as a teacher?") se muestra revelador en cuanto a los efectos positivos que este enfoque ha tenido en su trayectoria profesional y en su formación como docentes:

[P1] Lesson Study has opened a new way to teach a language in my practice. It has changed my focus and my view of students toward the subject.

[P2] This methodology has helped me experience new ways of collaborating and cooperating with colleagues, and also seeing firsthand how effective Cooperative Learning can be.

[P3] It was another template to follow among others. However, it keeps me organized when preparing my lesson.

[P4] Lesson Study has had a positive impact both in my professional career and in my training as a teacher, because I had never designed lesson plans collaboratively before. Now I think it is a useful approach because it can help to implement new classroom techniques. I will keep on reading on Lesson Study to implement it more frequently with my future pupils (if other teachers and colleagues are also willing to do that).

Analizaremos la discusión de nuestros resultados cuantitativos y cualitativos en el siguiente apartado.

\section{DISCUSIÓN Y CONCLUSIONES}

El análisis de los resultados de este estudio se muestra revelador en cuanto a las distintas comparaciones que se pueden establecer de los grupos de alumnos (primaria frente a secundaria; curso 18-19 frente a curso 20-21), y de estos en relación con los datos que ofrecen las entrevistas al profesorado.

En líneas generales podemos afirmar que el alumnado $(n=175)$ prefiere trabajar de manera cooperativa, ya que los datos de la Tabla 1 indican que los alumnos que señalan la opción de trabajar en grupos o por parejas 'normalmente' o 'siempre' suman el 78 \% para los alumnos del curso 18-19, y el $89 \%$ para los alumnos del curso 20-21. Esta ligera, pero a la vez significativa subida del porcentaje, es debida a que los estudiantes que repiten la experimentación de las LS durante el curso 20-21 (subgrupo de Primaria) indican en mayor medida que les gusta trabajar en grupo 'normalmente' o 'siempre'. Resulta significativo también que el resultado para 'nunca' es $0 \%$ para ambos subgrupos, Primaria y Secundaria. Estos resultados se alinean con los estudios de Decker, Calo, Yao y Weer (2015); Shaw, Duffy y Stark (2000); y Stark, Shaw y Duffy (2007), en los que se afirma que el alumnado tiene preferencia por el trabajo en grupo. 
Por otro lado, los resultados de la Tabla 2 revelan interesantes comparaciones que pueden establecerse entre el alumnado de Educación Primaria y Secundaria. El subgrupo de Primaria (curso 18-19) indica que lo que más le gusta del trabajo en grupo es, en orden de preferencia: "Ayudar a otros a aprender juntos" (44) y "Ayudar a sus compañeros a aprender cosas nuevas" (38). Este subgrupo de alumnos señala las mismas preferencias durante el curso 20-21, coincidiendo la puntuación para ambos ítems (33). Es de señalar que estos alumnos han experimentado las LS durante dos cursos en la misma asignatura (inglés en $3^{\circ}$ de Ed. Primaria durante el curso 18-19, e inglés en $5^{\circ}$ de Ed. Primaria durante el curso 20-21), y en ellos destaca una actitud y un comportamiento de ayuda a otros en sus sesiones de AC que, según los resultados del estudio de Dzemidzic Kristiansen (2020), resultan altamente beneficiosos para su propio aprendizaje.

El ítem que este subgrupo destaca en tercer lugar es "Charlar con mis compañeros sobre el trabajo que vamos a hacer" (puntuando 37 para el curso 18-19) y "Trabajar de forma diferente a la habitual" junto con "Hacer amigos/as nuevos/as" (puntuando 25 ambos ítems para el curso 20-21). Estos elementos indican una marcada preferencia por los aspectos sociales del aprendizaje cooperativo, además del gusto por compartir y comunicarse durante el desarrollo de las tareas. En este sentido, Johnson y Johnson (2002: 9) afirman: "Cooperative learning exists when students work together to achieve joint learning groups", dando cuenta de la vertiente social del aprendizaje que se lleva a cabo en grupo, tal como los datos de nuestro estudio señalan.

El subgrupo de Educación Secundaria (curso 18-19), por su parte, coincide con el subgrupo de Primaria (de ambos cursos) en su preferencia por "Ayudar a otros a aprender juntos" (35), pero coloca en la segunda posición de sus gustos "Trabajar de forma diferente a la habitual" (32). Estas valoraciones no cambian significativamente para el subgrupo de Educación Secundaria (curso 20-21), donde vuelven a destacar los mismos ítems, a pesar de que se trata de dos subgrupos de alumnos diferentes. Estos datos refuerzan el valor que este subgrupo de alumnos atribuye a la actitud de ayuda del alumnado en aprendizaje cooperativo, que habían señalado los alumnos del subgrupo de Educación Primaria (ambos cursos). Sin embargo, el subgrupo de Educación Secundaria (curso 20-21) coloca en segunda posición de sus preferencias el escape de la rutina en el trabajo habitual. Estos resultados se alinean con el estudio de Evans, Klymchuk, Murphy, Novak, Stephens y Thomas (2020), que asocian la aplicación de técnicas de aula que escapan de la rutina con el compromiso de los estudiantes con su propio aprendizaje.

El subgrupo de Educación Secundaria (curso 18-19) señala en tercera posición de sus preferencias dos ítems que coinciden en puntuación (18): i. "Charlar con mis compañeros/as sobre el trabajo que vamos a hacer" y ii. "Hacer amigos nuevos". La tercera posición de las preferencias de este subgrupo (curso 20-21) está compartida entre los ítems "Hacer amigos nuevos" y "Charlar con mis compañeros sobre el trabajo que vamos a hacer", alcanzando ambos ítems una puntuación de 18. De nuevo, este subgrupo subraya el carácter social del aprendizaje cooperativo que ya veíamos en el subgrupo de Educación Primaria.

Podemos afirmar, por tanto, que los datos de este trabajo aportan pruebas de que la adopción de prácticas de AC hace que el alumnado valore positivamente el trabajo en equipo, la capacidad de liderazgo (por ejemplo, ayudando a otros a aprender juntos) y el 
pensamiento crítico (que demuestra cuando afirma que quiere salir de la rutina). Todos estos beneficios han sido identificados por la literatura y nuestro trabajo abunda en estos datos, que remarcan el carácter social de las LS.

Los datos cualitativos de este trabajo, por otro lado, indican que ninguno de los 4 docentes conocía las LS con anterioridad y que su implementación (además de los seminarios formativos que se han llevado a cabo en el marco del proyecto) ha contribuido enormemente a la mejora de su formación como profesores por diversos motivos. El profesor 1 (P1) indica que las LS han cambiado su visión del alumnado y el enfoque de la asignatura que imparte, resultados que se alinean con el estudio de Lenski y Caskey (2009: 51), quienes afirman que "In these communities, teachers construct, organize, share, and refine their knowledge of the lesson." Las LS también son consideradas por los profesores participantes de este estudio como una forma positiva de colaborar con sus compañeros (P2 y P4), porque los ayuda a mejorar en la adaptación de diversas técnicas de enseñanza en el aula (P4) y a preparar sus clases (P3), resultados que confirman los hallazgos de Schipper, van der Lans, de Vries, Goei y van Veen (2020). Finalmente, las LS también son consideradas una influencia positiva en la formación permanente del profesorado y en su carrera profesional por el profesor no. 4 de nuestro trabajo (Cajkler, Wood, Norton, \& Pedder, 2013; Perry \& Lewis, 2009).

Por tanto, nuestras conclusiones finales se centran en dos cuestiones fundamentales. Por un lado, queremos subrayar los beneficios de las LS para el alumnado y el carácter social del aprendizaje que ellos reconocen en su implementación. Por otro lado, nos gustaría destacar la recomendación de que el profesorado implemente en su aula las LS con frecuencia, tal como indican los resultados de esta investigación, ya que la importancia de estructurar el trabajo cooperativo beneficia el aprendizaje (Sulfemi \& Kamalia, 2020) y puede contribuir a mejorar la formación (inicial y permanente) del profesorado, además de que mejora la adopción de técnicas adaptativas en el aula y la preparación de las clases, en línea con los resultados de Schipper, van der Lans, de Vries, Goei, y van Veen (2020). Además, los beneficios que las LS reportan para el alumnado se localizan en distintas áreas del aprendizaje, tal como indican los resultados de este trabajo, lo que supone una cualidad añadida de las LS al proceso educativo.

\section{REFERENCIAS BIBLIOGRÁFICAS}

Cajkler, W., Wood, P., Norton, J., \& Pedder, D. (2013). Lesson study: towards a collaborative approach to learning in initial teacher education?. Cambridge Journal of Education, 43(4), 537554. https://doi.org/10.1080/0305764x.2013.834037.

Campo-Arias, A., \& Oviedo, H. C. (2008). Psychometric properties of a scale: Internal consistency. Revista de salud pública, 10(5), 831-839. https://doi.org/10.1590/s0124-00642008000500015.

Creswell, J. W., \& Poth, C. N. (Eds.). (2018). Qualitative inquiry and research design. Choosing among five traditions $\left(4^{\text {th }} \mathrm{ed}\right.$.). Londres: SAGE.

Decker, W. H., Calo, T. J., Yao, H., \& Weer, C. H. (2015). Preference for group work in China and the US. Cross Cultural Management, 22(1), 90-115. https://doi.org/10.1108/CCM-03-2013-0053. 
Dzemidzic Kristiansen, S. (2020). Exploring pupils' and teachers' perspectives on faceto-face promotive interaction in cooperative learning. Education, 3(13), 1-16. https://doi.org/10.1080/03004279.2020.1833060.

Estrella, S., Zakaryan, D., Olfos, R., \& Espinoza, G. (2019). How teachers learn to maintain the cognitive demand of tasks through Lesson Study. Journal of Mathematics Teacher Education, 23, 293-310. https://doi.org/10.1007/s10857018-09423-y.

Evans, T., Klymchuk, S., Murphy, P. E., Novak, J., Stephens, J., \& Thomas, M. (2020). Engagement of undergraduate STEM students: the influence of non-routine problems. Higher Education Research \& Development, 1-17. https://doi.org/10.1080/07294360.2020.1835838.

Guner, P., \& Akyuz, D. (2020). Noticing Student Mathematical Thinking within the Context of Lesson Study. Journal of Teacher Education, 71(5), 568-583. https://doi.org/10.1177/0022487119892964.

Hasan, R. M., Lukitasari, M., \& Ernawati, Y. (2021). Students' achievement and teachers' perception in the implementation of lesson study-based cooperative learning. Journal of Physics: Conference Series 1731012004. https://doi.org/10.1088/1742-6596/1731/1/012004. https://cutt.ly/XbEu0SH.

Johnson, D. W., \& Johnson, R. T. (2002). Cooperative learning and social interdependence theory. En R. Scott Tindale et al. (Eds.), Theory and research on small groups, 9-35. Springer.

Kyprianidou, M., Demetriadis, S., Tsiatsos, T., \& Pombortsis, A. (2012). Group formation based on learning styles: can it improve students' teamwork?. Educational Technology Research and Development, 60(1), 83110. https://doi.org/10.1007/s11423-011-9215-4.

Lenski, S. J., \& Caskey, M. M. (2009). Using the Lesson Study Approach to Plan for Student Learning. Middle School Journal, 40(3), 50-57. https://doi.org/10.1080/00940771.2009.11495587.

Peña-Trapero, N., \& Pérez-Gómez, A. I. (2017). Pedagogical potentialities of lesson study for the reconstruction of teachers' dispositions. International Journal for Lesson and Learning Studies, 6(1), 66-79. https://doi.org/10.1108/IJLLS-092016-0029.

Perry, R. R. \& Lewis, C. C. (2009). What is successful adaptation of lesson study in the US? Journal of Educational Change, 10, 365-391. https://doi.org/10.1007/s10833-008-9069-7.

Schipper, T. M., van der Lans, R. M., de Vries, S., Goei, S. L., \& van Veen, K. (2020). Becoming a more adaptive teacher through collaborating in Lesson Study? Examining the influence of Lesson Study on teachers' adaptive teaching practices in mainstream secondary education. Teaching and Teacher Education, 88, 102961. https://doi.org/10.1016/j.tate.2019.102961.

Shaw, J. D., Duffy, M. K., \& Stark, E. M. (2000). Interdependence and preference for group work: Main and congruence effects on the satisfaction and performance of group members. Journal of Management, 26(2), 259279. https://doi.org/10.1177/014920630002600205.

Shi, W., \& Han, L. (2019). Promoting Learner Autonomy through Cooperative Learning. English Language Teaching, 12(8), 30-36. https://doi.org/10.5539/elt.v12n8p30.

Stark, E. M., Shaw, J. D., \& Duffy, M. K. (2007). Preference for group work, winning orientation, and social loafing behavior in groups. Group \& Organization Management, 32(6), 699-723. https://doi.org/10.1177/1059601106291130. 
Stigler, J. W. (2004). Foreword. En C. Fernández \& M. Yoshida (Eds.), Lesson Study. A Japanese Approach to Improving Mathematics Teaching and Learning, ix-xi. Lawrence Erlbaum Associates.

Sulfemi, W. B., \& Kamalia, Y. (2020). Jigsaw Cooperative Learning Model Using Audiovisual Media to Improve Learning Outcomes. JPsd (Jurnal Pendidikan Sekolah Dasar), 6(1), 30-42.

https://jurnal.untirta.ac.id/index.php/jpsd/article/viewFile/4919/5063

Uştuk, Ö., \& De Costa, P. I. (2020). Reflection as meta-action: Lesson study and EFL teacher professional development. TESOL Journal, e531. https://doi.org/10.1002/tesj.531. https://cutt.ly/4bEt9LA

Wahyukti, T. (2017, October). Enhancing Students' Cooperative Learning in an EFL Classroom through Lesson Study. En $4^{\text {th }}$ Asia Pacific Education Conference (AECON 2017, 283-287. Atlantis Press.

Widita, A., \& Nurihsan, A. J. (2020). The Development of Internalization of Character Responsibility through the Cooperative Learning Model on Students in Elementary School. International Conference on Elementary Education, 2(1), 387-394. http://proceedings.upi.edu/index.php/icee/article/view/640/555.

Wood, P., \& Cajkler, W. (2018). Lesson study: A collaborative approach to scholarship for teaching and learning in higher education. Journal of Further and Higher Education, 42, 313-326. https://doi.org/10.1080/0309877X.2016.1261093. 